INVESTIGACIÓN DIDÁCTICA

\title{
Los observadores ante el ciberacoso (cyberbullying)
}

\section{Blanca González Arévalo}

RESUMEN: El objetivo de este estudio es conocer la prevalencia de observación de ciberacoso en escolares de Educación Secundaria Obligatoria (ESO), y el comportamiento o reacciones que tienen ante este fenómeno. Se trata de un estudio transversal, descriptivo y observacional en dos centros escolares de Educación Secundaria Obligatoria (ESO) de Toledo. Se estudian 190 alumnos y alumnas de 11 a 16 años. Se utilizó el CCB. Cuestionario de Cyberbullying de Garaigordobil y Fernández-Tomé. El 74,2\% de los alumnos han observado algunas de las formas de este tipo de acoso como el robo de contraseña, emisión de mensajes ofensivos a través de móvil y/o internet. Los alumnos que observan este tipo de acoso lo comentan antes a sus compañeros que a los adultos. Las estrategias para luchar contra el ciberacoso pueden dirigirse también hacia los observadores de este tipo de conductas.

Palabras clave: Ciberacoso; Observadores; Efectos; Adolescentes; Tecnologías de la información y la comunicación (TIC); Acoso escolar.

\section{ABSTRACT: observers of cyberbullying}

The aim of this study is to know the prevalence of observation of cyberbullying among Secondary School students as well as their behavior and response when facing this situation. We designed a transversal, descriptive and observational study in two Secondary School students in Toledo (Spain). The sample comprised 190 students of both genders and ages between 11 and 16 years. It was used the CCB. Questionnaire of cyberbullying from Garaigordobil y Fernández-Tomé. Results indicated that 74, 2\% of them have observed someone of the forms of this type of harassment such as stealing the password, sending offensive messages through the mobile phone or internet. The students that observe this type of harassment, tell their classmates before adults. We can focus the strategies for fighting against cyberbullying towards the observers too.

Keywords: Cyberbullying; Observers; Effects; Teenagers; Information and communication technology (ICT); School harassment.

$* * * *$

\section{RÉSUMÉ: les observateurs face au cyber-harcèlement}

L'objectif de cet étude est de connaître l'importance de l'observation du cyber-harcèlement et du comportement de ces élèves, dans deux Lycées de Tolède. L'échantillon comprenait 190 élèves des deux sexes et d'âges entre 11 et 16 ans. II a été utilisé le CCB: Questionnaire de la Cyberbullying de Garaigordobil y Fernández-Tomé. Cent quatre-vingt-dix élèves, garçons et filles ont participé dans l'étude. $74,2 \%$ des élèves ont observé quelques formes de ce type de harcèlement comme le vol du mot de passe, l'émission des messages offensifs á travers du portable et/ou d'Internet. Les élèves qui observent ce tipe de harcèlement en parlent avant avec leurs camarades qu'avec les adultes. Les stratégies pour combattre le cyber-harcèlement peuvent aussi être adressées vers les observateurs de ce type de conduite..

Mots clé: Harcèlement sur la net; Observateur; Effets; Adolescents; Technologies de l'information et de la communication (TIC); Harcèlement á l'école.

\footnotetext{
* Dirección: C/ Trigal 73 Urb. Las Perdices. 45593 Bargas. Toledo. Teléfono: 609220876

E-mail: blanca.institituto@gmail.com.

1 El término observador se utiliza en este artículo como sinónimo de testigo o espectador: "Persona que se percata del abuso o del acto negativo ejercido en contra de otra."
}

$\triangle$ Artículo recibido el 26 de Mayo de 2013 y aceptado el 1 de Junio de 2015. 


\section{Introducción}

El ciberacoso es el uso de los medios telemáticos (Internet, telefonía móvil y videojuegos online principalmente) para ejercer acoso psicológico. El desarrollo de las Tecnologías de la Información y Comunicación (TIC) supone una oportunidad inimaginable hasta hace sólo unos pocos años para tener acceso al conocimiento e intercambiarlo con personas al otro lado del mundo casi al instante, ofreciendo una gran oportunidad para los educadores. Sin embargo, también tienen aspectos negativo que se han desarrollado vertiginosamente de forma paralela a los aspectos positivos que hemos comentado. Uno de ellos es el ciberacoso escolar entre iguales. A diferencia del Bullying, que se produce fundamentalmente en el centro educativo, el ciberacoso, al tratarse de una forma de acoso indirecto y no presencial, se puede realizar fuera de él; el agresor no tiene contacto con la víctima, no ve su cara, sus ojos, su dolor, su pena, con lo cual difícilmente podrá llegar a empatizar o despertar su compasión por el otro. Sin embargo, no es fácil la detección de este problema, porque el niño acosado a veces se siente culpable o tiene miedo a que sus padres o cuidadores tomen medidas que puedan perjudicarle, como quitarle el teléfono o impedirle el acceso a internet y oculta el problema.

Conocer los aspectos personales de la relación agresor-víctima es importante, pero ello sólo se refiere a una parte del problema. La intimidación implica a más personas que las directamente afectadas, ya que se vive dentro de un grupo de compañeros que adoptan una serie de roles, ya sea como agentes activos, como víctimas, como observadores como defensores, y que pueden experimenta una gran variedad de emociones. Los autores casi nunca actúan solos, sino que, por lo general, lo hacen con el apoyo de su círculo inmediato de asistentes y reforzadores (Salmivalli et al., 1996). El acoso se intensifica aún más como resultado de las respuestas de los que estaban allí como observadores tanto si reaccionan con indiferencia a la difícil si- tuación de la víctima como si implícitamente aprueban lo que está sucediendo. Sólo una pequeña proporción de observadores actuará en el papel de los defensores y ofrecerán apoyo emocional y protección a las víctimas. Los observadores se sienten atrapados en un "dilema social": a pesar de ser conscientes de que el acoso está mal y que deberían intentar detenerlo, son plenamente conscientes de sus propias necesidades de seguridad y de protección dentro del grupo de compañeros. Por desgracia, a través de su inacción, los espectadores no sólo refuerzan la conducta de acoso sino que también amenazan la necesidad de la víctima de pertenecer al grupo de iguales (Salmivalli, 2010)

Los resultados empíricos muestran que las cibervíctimas tienden a pedir más ayuda a sus amigos o compañeros que a sus padres o a los maestros (Aricak et al., 2008). Muchos de los estudiantes admiten que hablarían con sus compañeros sobre el ciberacoso e investigaciones previas sugieren que los compañeros pueden ayudar a combatir y prevenir este fenómeno, en particular, los partidarios del acosador y los observadores (DiBasilio, 2008). Cuando se les preguntó a estudiantes sobre cuál sería su reacción si sufrieran ciberacoso, respondieron que lo más probable sería que acudiesen a buscar ayuda de los amigos (Wright et al., 2009). Por lo tanto, cualquier intervención que se realice para controlar este problema deberá tener en cuenta estas consideraciones y funcionará mejor si se integra en la política global de la escuela y se dirige a todos los agentes que pueden participar de algún modo en estas conductas: padres, profesores, amigos, acosador y acosado, incluyendo, no sólo medidas disciplinarias cuando ya haya ocurrido el problema, sino formación específica en habilidades sociales para impedirlas.

En este trabajo nos propusimos analizar las características de la conducta de uno de estos agentes "participantes" en el ciberacoso, los observadores, considerando que este es el primer paso para elaborar estrategias de prevención y control de estas formas de actuar en las que ellos puedan participar.

\section{Material y métodos}

\section{Diseño y ámbito de estudio}

Se trata de un estudio observacional descriptivo y transversal realizado en el ámbito escolar de dos centros educativos de Toledo, uno público y otro privado-concertado, en el periodo comprendido entre noviembre de 2011 y febrero de 2012. De los 9 centros educativos públicos y de los 8 centros privado-concertados, se escogieron al azar uno público y otro privado. Se solicitó permiso por carta a la Consejería de Educación y a la Dirección de cada uno de los dos centros educativos y se explicó, tanto a los equipos de orientación pedagógica como las direcciones de los centros, en qué consistí la investigación, cuáles eran los objetivos de estudio y el diseño del cuestionario que iba a ser cumplimentado por los alumnos.

\section{Población de estudio}

Los participantes en este estudio son lo alumnos de $1^{\circ}$ y $3^{\circ}$ de Educación Secundaria Obligatoria (ESO) de los dos centros seleccionados. La muestra final la componían 190 alumnos. Como criterio de exclusió únicamente se consideró la negativa a partici par en el estudio.

\section{Variables estudiadas}

Las variables de estudio han sido agrupadas en cuatro apartados: Variables socio-demográficas en las que se incluyen: edad, género, curso y centro educativo. Variables de uso TIC: ubicación del ordenador en casa, acceso a internet y teléfono móvil propio. Variable del estudio relacionadas con la observación del acoso: Prevalencia de observación de acoso conductas observadas de ciberacoso, intensidad de las mismas, pertenencia al grupo acosador, grado de acuerdo con lo que se observa, empatía con las víctimas, acciones que reali- za y sentimientos que tiene, al observar estas conductas. Y variables del estudio relacionadas con las estrategias de afrontamiento y acciones propuestas por los escolares para evitar el ciberacoso.

\section{Instrumento de medición}

Para la medición de las variables sociodemográficas y generales se diseñó una ficha de recogida de datos que se incorporó al cuestionario principal.

El instrumento utilizado para la medición de las variables del estudio ha sido el CCB (Cuestionario de Cyberbullying) de Garaigordobil y Fernández-Tomé, (2011). No es un instrumento adaptado, es original. Los coeficientes alfa de Cronbach para la sección de cyberbullying (total $?=0.91$; cibervictimización $?=0.82$ ciberobservación $?=0.87$ ), evidenciaron adecuada consistencia interna. Los coeficientes Gamma en los indicadores de cyberbullying (entre 0.60 y 0.81 ) confirman una estabilidad temporal adecuada. Respecto a la validez, queremos destacar que correlaciona positiva y significativamente con otras medidas de acoso escolar, y, de forma convergente con resolución agresiva de conflictos y conductas antisociales. Este cuestionario permite identificar Cyberbullying y evalúa 15 conductas de $\mathrm{CB}$ para identificar víctimas y observadores. En relación a los observadores de CB: pertenencia al grupo acosador, grado de acuerdo con lo que se observa, empatía con las víctimas, acciones que se realizan, sentimientos que tiene al observar esas conductas, identificar las estrategias de afrontamiento disponibles en los adolescentes y acciones que deben desarrollar las víctimas y los observadores.

\section{Recogida y análisis estadístico} de los datos

Los cuestionarios fueron pasados a los alumnos durante el horario escolar por sus propios profesores, explicándoles que su participa- 
ción en el estudio era totalmente voluntaria, individual y anónima. Los datos fueron tratados respetando la confidencialidad y el anonimato de los participantes, y bajo las consideracione legales vigentes actualmente en nuestro país (ley de protección de datos). Para su manipulación y ordenación fueron introducidos en una base de datos de Excel y para su análisis estadístico se utilizó el programa estadístico Statistical Package for the Social Sciences (SPSS v.12) en español.

\section{Resultados}

\section{Resultados descriptivas de variables} socio-demográficas y generales

De los 190 alumnos con una edad media de 13,35 (DT 1,27) años, $116(61,4 \%)$ son mujeres y $74(38,6 \%)$ son varones; $96(50,5 \%) \mathrm{d}$ los alumnos corresponden a un centro educativo de titularidad pública y $94(49,5 \%)$ al centro de titularidad privada; 99 alumnos $(52,1 \%)$ están matriculados en $3^{\circ}$ de ESO y 91 $(47,9 \%)$ en $1^{\circ}$ de ESO. De la muestra total de alumnos, $176(93,1 \%)$ tienen teléfono móvil, $174(94,6 \%)$ tienen conexión a Internet y 82 de ellos $(43,4 \%)$ tienen el ordenador en su habitación (Ver Tabla 1).

\begin{tabular}{|c|c|c|c|c|}
\hline & $\begin{array}{l}1^{\circ} \text { ESO } \\
\text { COLEGIO } \\
\text { PÚBLICO }\end{array}$ & $\begin{array}{l}3^{\circ} \text { ESO } \\
\text { COLEGIO } \\
\text { PÚBLICO }\end{array}$ & $\begin{array}{l}1^{\circ} \text { ESO } \\
\text { COLEGIO CONCERTADO/ } \\
\text { PRIVADO }\end{array}$ & $\begin{array}{l}3^{\circ} \text { ESO } \\
\text { COLEGIO CONCERTADO/ } \\
\text { PRIVADO }\end{array}$ \\
\hline No de alumnos & 50 & 46 & 41 & 53 \\
\hline Edad media & 12,16 & 14,30 & 12,21 & 14,50 \\
\hline Género & $18 \mathrm{~V} / 32 \mathrm{M}$ & $26 \mathrm{~V} / 20 \mathrm{M}$ & $15 \mathrm{~V} / 26 \mathrm{M}$ & $15 \mathrm{~V} / 38 \mathrm{M}$ \\
\hline $\begin{array}{l}\text { Conexión } \\
\text { a Internet }\end{array}$ & 45 & 41 & 39 & 50 \\
\hline Móvil & 45 & 44 & 36 & 51 \\
\hline $\begin{array}{l}\text { Ordenador en } \\
\text { su habitación }\end{array}$ & 22 & 22 & 16 & 22 \\
\hline
\end{tabular}

Tabla 1. Distribución de la muestra total por variables sociodemográficas y generales. Fuente: elaboración propia.

\section{Resultados de prevalencia e intensidad} de la observación del $\mathrm{CB}$

De los 190 alumnos, los resultados indican que $141(74,2 \%)$ han observado la existencia de este tipo de acoso. La edad media del alumno observador es de 13.12 (DT 1.09) años; 93 (66.4\%) de los observadores son mujeres y 47 (33.6\%) varones; 74 (52.2\%) pertenecen al centro educativo de titularidad concertada/privada y 67 (47.5\%) al público; 75 (53.2\%) pertenecen a $3^{\circ}$ de ESO y $66(46 \%)$ a $1^{\circ}$ de ESO.

De los 141 alumnos que han observado este tipo de acoso, 130 (92.2\%) lo han observado algunas veces, $10(7.1 \%)$ ha sido a menudo y 1 alumno $(0.7 \%)$ siempre ha observado conductas de ciberacoso.

\section{Resultados sobre conductas de CB} en observadores

Dentro de las conductas observadas, la de mayor frecuencia es el robo de contraseña, 73 $(39,9 \%)$, seguida de la emisión de mensajes ofensivos a través de móvil y/o internet, 56 (30,4\%), llamadas anónimas para asustar y provocar miedo, $49(26,6 \%)$ y difusión de rumores con el fin de dañar al acosado, 51 (28,0\%). De los observadores de CB, 21 (11,5\%) de ellos han visto agresiones grabadas y colgadas en internet (Ver Tabla 2).

\begin{tabular}{|c|c|c|c|c|}
\hline Conductas & Nunca & A VECES & A MENUdo & SIEMPRE \\
\hline $\begin{array}{l}\text { Mensajes ofensivos a través } \\
\text { de móvil y/o Internet }\end{array}$ & $119(64,7 \%)$ & $56(30,4 \%)$ & $6(3,3 \%)$ & $3(1,6 \%)$ \\
\hline $\begin{array}{l}\text { Llamadas ofensivas a través } \\
\text { de móvil y/o Internet }\end{array}$ & $143(78,1 \%)$ & $31(16,9 \%)$ & $6(3,3 \%)$ & $3(1,6 \%)$ \\
\hline $\begin{array}{l}\text { Agresión grabada y colgada } \\
\text { en Internet (happy slappin) }\end{array}$ & $158(86,3 \%)$ & $21(11,5 \%)$ & $3(1,6 \%)$ & $1(0,5 \%)$ \\
\hline $\begin{array}{l}\text { Difundir fotos privadas } \\
\text { o comprometidas a través } \\
\text { de móvil y/o Internet }\end{array}$ & $139(76,4 \%)$ & $38(20,9 \%)$ & $4(2.2 \%)$ & $1(0.5 \%)$ \\
\hline $\begin{array}{l}\text { Fotos robadas y colgadas } \\
\text { en Internet o difundidas } \\
\text { vía móvil }\end{array}$ & $153(83,6 \%)$ & $26(14,2 \%)$ & $4(2,2 \%)$ & $0(0 \%)$ \\
\hline $\begin{array}{l}\text { Llamadas anónimas para } \\
\text { asustar }\end{array}$ & $119(64,7 \%)$ & $49(26,6 \%)$ & $14(7,6 \%)$ & $2(1,1 \%)$ \\
\hline $\begin{array}{l}\text { Chantajes o amenazas } \\
\text { a través de mensajes } \\
\text { y/o llamadas }\end{array}$ & $154(84,2 \%)$ & $27(14,8 \%)$ & $1(0.5 \%)$ & $1(0,5 \%)$ \\
\hline $\begin{array}{l}\text { Acoco sexual a través del } \\
\text { móvil y/o Internet }\end{array}$ & $166(90,7 \%)$ & $11(6,0 \%)$ & $4(2,2 \%)$ & $2(1,1 \%)$ \\
\hline $\begin{array}{l}\text { Suplantación de la persona } \\
\text { para difamar, mentir } \\
\text { o contar tus secretos }\end{array}$ & $129(70,5 \%)$ & $41(22,4 \%)$ & $12(6,6 \%)$ & $1(0.5 \%)$ \\
\hline Robo de contraseña & $89(48,6 \%)$ & $73(39,9 \%)$ & $16(8,7 \%)$ & $5(2,7 \%)$ \\
\hline $\begin{array}{l}\text { Trucado de fotos o videos } \\
\text { para difundirlos y } \\
\text { humillarte en Internet }\end{array}$ & $152(83,1 \%)$ & $25(13,7 \%)$ & $4(2.2 \%)$ & $2(1,1 \%)$ \\
\hline $\begin{array}{l}\text { Acoso para aislarle de } \\
\text { sus contactos en las redes } \\
\text { sociales }\end{array}$ & $158(86,8 \%)$ & $18(9,9 \%)$ & $4(2,2 \%)$ & $2(1,1 \%)$ \\
\hline $\begin{array}{l}\text { Chantaje a cambio de } \\
\text { no divulgar información } \\
\text { intima }\end{array}$ & $162(88,5 \%)$ & $16(8,7 \%)$ & $3(1,6 \%)$ & $2(1,1 \%)$ \\
\hline $\begin{array}{l}\text { Amenazas de muerte a él } \\
\text { o su familia a través de } \\
\text { mensajes, llamadas y /o } \\
\text { Internet }\end{array}$ & $161(88,5 \%)$ & $17(9,3 \%)$ & $2(1,1 \%)$ & $2(1,1 \%)$ \\
\hline $\begin{array}{l}\text { Difamar o difundir } \\
\text { rumores para hacer daño }\end{array}$ & $115(63,2 \%)$ & $51(28,0 \%)$ & $10(5,5 \%)$ & $6(3,3 \%)$ \\
\hline
\end{tabular}

Tabla 1. Conductas de CB observadas manifestadas por observadores en frecuencia y porcentaje. Fuente: elaboración propia. 


\section{Resultados sobre cómo actúan}

los observadores cuándo ven o saben

que están ocurriendo estas situaciones

De los 190 alumnos solo 50 (26\%) responden a la pregunta. De estos: 34 (68\%) no hacen nada $5(10 \%)$ refieren estar de acuerdo con el acoso, 11 (22\%) consideran que las víctimas se lo merecen.

\section{Resultados sobre a quién comunican los observadores este tipo de acoso}

De los 190, responden a la pregunta 12 (63.68\%); de estos 65 (53.72\%) lo comunican a sus compañeros, $40(33.06 \%)$, lo comunican a sus padres, $12(9.92 \%)$ a sus profesores y 4 (3.30\%) no lo comunican a nadie (Ver Tabla 3 ).

\section{Resultados sobre sentimientos más} frecuentes frente al CB en observadores

Dentro de los sentimientos que provocan este tipo de conductas en el grupo de los observadores, la preocupación ocupa el primer lugar seguido de tristeza (Ver Tabla 4).

\section{Resultados sobre las propuestas que hacen observadores y víctimas de $C B$ para evitarlo}

Como medidas preventivas frente al $\mathrm{CB}$ destacan la comunicación del problema a los adultos en el caso de que fuesen acosados y el apoyo a las víctimas en el caso de que observasen conductas de CB (Ver Tabla 5).

\begin{tabular}{|c|c|c|c|}
\hline Compañeros & Padres & Profesores & A nadie \\
\hline $65(53.72 \%)$ & $40(33.06 \%)$ & $12(9.92 \%)$ & $4(3.30 \%)$ \\
\hline
\end{tabular}

Tabla 3. Comunicación de CB por parte de los observadores. Fuente: elaboración propia.

\begin{tabular}{|c|c|c|c|c|c|c|c|c|}
\hline Preocupación & Tristeza & Rabia & Miedo & Rencor & Culpa & Indiferencia & Vergüienza & Venganza \\
\hline $29,46 \%$ & $25,73 \%$ & $18,26 \%$ & $7,88 \%$ & $6,64 \%$ & $4,98 \%$ & $4,15 \%$ & $2,90 \%$ & $0 \%$ \\
\hline
\end{tabular}

Tabla 4. Sentimientos más frecuentes en observadores de CB. Fuente: elaboración propia.

\begin{tabular}{|l|c|c|}
\hline Actitudes & Acosados & Observadores \\
\hline Comunicar adultos & $70(58,8 \%)$ & $35(29,9 \%)$ \\
\hline Denunciar a la policía & $31(26,1 \%)$ & $17(14,5 \%)$ \\
\hline Ignorar acoso & $9(7,6 \%)$ & $0(0 \%)$ \\
\hline Mediar con el acosador & $0(0 \%)$ & $11(9,4 \%)$ \\
\hline Pedir ayuda & $4(3,4 \%)$ & $0(0 \%)$ \\
\hline Otras & $5(4,2 \%)$ & $14(12,0 \%)$ \\
\hline
\end{tabular}

Tabla 5. Actitudes propuestas por los alumnos como actividades preventivas frente al $\mathrm{CB}$. Fuente: elaboración propia.
Resultados sobre diferencias

de observación de CB según titularidad del centro, curso y género

No se aprecian diferencias estadísticamente significativas en cuanto a la observación de $\mathrm{CB}$ según los alumnos matriculados en $1^{\circ} \mathrm{de}$ ESO con un $46,8 \%$ y aquellos que están matriculados en $3^{\circ}$ de ESO con un 53,2\%; ( $\left.p=0,611\right)$ ni entre los dos centros. Para finalizar, hemos podido comprobar la existencia de diferencias estadísticamente significativas en cuanto a la observación de acoso entre el grupo de mujere con un $66,4 \%$ frente al grupo de hombres con un $33,6 \%$; $(\mathrm{p}=0.016)$

\section{Discusión de los resultados}

La mayoría de los estudios que se han hecho sobre ciberacoso han ido dirigidos fundamentalmente a conocer la prevalencia de este tipo de acoso y a conocer las características de lo acosadores y de las víctimas. Hay menos estudios dirigidos a conocer los diferentes aspecto de este tema relacionados con las personas que observan estas conductas, aunque en mucho casos no las hayan practicado o sufrido. No propusimos en este trabajo analizar algunas características de los escolares que han observad en alguna ocasión conductas de ciberacoso; conocimiento de este tipo de datos quizás pueda contribuir a ampliar el campo hacia el que s puedan enfocar diferentes estrategias para luchar contra este creciente problema. Los resultados han sido extraídos de un trabajo más am plio en el que se analizaban también aspectos relacionados con las víctimas y los acosadores.

Hemos podido comprobar que el uso de las TIC en la población estudiada arroja cifra que no son muy diferentes a las publicadas por el INE (Urueña, 2011), aunque con un porcentaje mayor de niños con teléfono móvil propio (93\% frente a 65\%). Estas diferencias pueden ser explicadas por los diferentes rangos de edad escogidos. La encuesta del INE incluye niños de 10 y 11 años, que son los subgrupos en los que los porcentajes bajan más. El uso de esta nuevas formas de comunicación, por lo tanto, se ha extendido rápidamente en nuestro país, como en el resto del mundo y en muchas ocasiones sin claras normas sobre su buen uso o sin la difusión necesaria para el adecuado conocimiento de quienes las usan, sobre todo de los niños.

Llama la atención el hecho de que casi el $75 \%$ de los alumnos entrevistados hayan observado en alguna ocasión conductas de ciberacoso, aunque la mayoría lo ha hecho sólo ocasionalmente. Sin embargo, más de un 7\% refieren haberlas observado a menudo. Esta cifra puede parecer pequeña, pero si extrapolamos estos datos a la población general de niños españoles en esta edad, obtendríamos datos realmente preocupantes. Estos resultados pueden estar indicándonos que los niños consideran estas conductas "lo normal" por lo frecuentes que son.

No encontramos diferencias entre los dos colegios ni entre los cursos, pero sí hubo diferencias estadísticamente significativas en cuanto al género. Las niñas dijeron haberlo observado más frecuentemente $(66.4 \%)$ que los niños (33.6\%). No podemos saber si esto es consecuencia de que las niñas comparten más la información o de que son más colaboradoras a la hora de contestar las encuestas.

Si analizamos los datos obtenidos acerca de las diferentes conductas de ciberacoso observadas encontramos una amplia variedad; desde las que pueden parecer menos problemáticas a priori como enviar mensajes ofensivos, hasta las que podríamos considerar como muy graves como acosar sexualmente a través del móvil Que más del 50\% de los alumnos entrevistados hayan observado robo de la contraseña a algún compañero puede significar que no son conscientes de lo que significa una contraseña ni los que la roban ni los que la poseen y deberían custodiarla mejor para evitar que otras personas puedan acceder a su información personal.

Sorprende que sólo 50 alumnos de los 190 entrevistados (26\%), respondan a la pregunta de cómo actúan cuando observan estas conductas. Como escribimos más arriba parece que esto es "lo normal" y por lo tanto no merece la pena ni contestar a esta pregunta. Pero de los pocos 
alumnos que contestaron, la mayoría, no hace nada e incluso algunos están de acuerdo con estas conductas o consideran que los compañero agredidos se lo merecen. Interpretamos, por un lado, que no son conscientes del peligro de estas acciones y que nadie les ha enseñado cómo deben actuar cuando tienen conocimiento de ellas y, por otro, la actuación del observador va a depender en gran medida del rol que adopte frente al acoso que ve: a) como observador indiferente, b) como observador que disfruta del acoso, c) como observador que no está de acuerdo con el acoso y que puede estar molesto (Mendoza, 2012)

El hecho de que más de la mitad de los que observan estas conductas lo comuniquen a sus compañeros antes que a sus padres o profesores puede estar indicándonos que no le dan much importancia al problema y que es una anécdota más de la vida diaria de un escolar del siglo XXI para comentar en los momentos de charla Contrasta, sin embargo, que estas conductas le provoquen un amplio abanico de sentimientos, pero que no les conduzcan a ninguna acción positiva, aunque opinen que la comunicación a un adulto sería la mejor medida para solucionar el problema o prevenir que aumente su gravedad. Es obvio que los efectos más acusados se muestran en la víctima, pero los observadores también son receptores de aprendizajes y hábitos negativos que influirán en su comportamiento actual y futuro. Todos los implicado en situaciones de maltrato, en cualquiera de los roles, están en mayor situación de riesgo de sufrir desajustes psicosociales y trastorno psicopatológicos en la adolescencia y en la vida adulta, que los chicos y chicas no implicados (Garaigordobil, 2011).

Creemos que nuestros escolares no son conscientes, en general, de lo grave que puede llegar a ser este tipo de acoso y de lo vulnerable que son cuando utilizan estas tecnologías de forma inadecuada. Lo que empieza siendo un juego puede acabar siendo un drama. Aunque preocupa a la sociedad la repercusión que esta conductas puedan producir de forma aguda en el momento de sufrirlas o realizarlas un niño, cabe pensar que las consecuencias a medio o largo plazo puedan ser peores. El daño psicológico que puede tener una víctima de ciberacoso es mayor que el producido por otros tipos de acoso. El hecho de que una información o imagen lesiva pueda colgarse en internet y ser accesible para otros durante largos periodos de tiempo, haciendo que el daño sea continuo y difícil de evitar por parte de la víctima, le da a esta forma de acoso unas connotaciones especiales que pueden ser muy perjudiciales; es por ello que los observadores de estas conductas no pueden ni deben quedarse al margen, como espectadores de este tipo de acoso; juegan un papel muy importante, ya que pueden frenar el acoso apoyando a las víctimas y denunciando a los agresores. Quizás sea más fácil en muchos casos obtener información de los observadores, meros espectadores de los hechos, que no de las víctimas que por diversas razones pueden ocultarlos y empeorar su gravedad y por supuesto de los que acosan.

Algunos de los resultados obtenidos en este estudio nos pueden estar indicando que nadie les ha informado de este riesgo, de lo importante que es utilizar bien estas tecnologías y saber identificar las situaciones de acoso para defenderse o ayudar a otros si lo necesitan. Tal como se refleja en el Protocolo de actuación escolar ante el ciberacoso del Equipo Multidisciplinar de Investigación sobre Cyberbullying (EMICI, 2012), el papel de la "audiencia" es de suma importancia ante este tipo de acoso ya que los espectadores pueden convertirse transitoriamente en colaboradores. Existen personas que sin ser agresores o víctimas, pueden verse implicadas de forma indirecta, bien apoyando a quien agrede o por el contrario, intentando ayudar a la víctima del ciberacoso.

Este trabajo tiene algunas limitaciones, entre otras, el pequeño tamaño de la muestra, el quedar circunscrito a una ciudad y el hecho de que a los alumnos se les pasó el cuestionario sin darles ninguna información previa; esto puede haber provocado que algunas preguntas no se hayan entendido bien, pero también creemos tas evitando que algunas respuestas puedan ser inducidas y crear un sesgo.
Como conclusión, las TIC son utilizadas con frecuencia por nuestros escolares. El ciberacoso es un fenómeno frecuente y creciente en nuestro medio. Los padres y los colegios deben tener información periódica sobre los nuevos hallazgos que se detecten. Considerando que no podemos ni debemos evitar el uso de las tecnologías por parte de los alumnos, la mejor manera de evitar sus efectos nocivos es enseñándoles a utilizarlas bien. No olvidemos que la escuela, además de su importante labor de enseñanza y de formación académica, también ha sido siempre un centro de formación humana y cívica, que prepara al individuo para saber vivir en sociedad, en base a unos principios morales, como el respeto y la solidaridad. Hoy en día este civismo se debe llevar al uso de las tecnologías de la información y comunicación, puesto que representan la nueva forma de socialización de los más jóvenes. En esta línea, materias como "Cuidado de la identidad digital", "Desarrollo de relaciones en línea, "Ciber-ciudadanía" deberían ser incorporadas a la enseñanza desde la educación primaria.

Los padres y educadores deben estar preparados para detectar precozmente en sus hijos o alumnos signos que puedan hacernos pensar que un chico o chica pueda estar sufriendo ciberacoso y, si lo niega, poder contac tar con personas preparadas para detectarlo. Es necesario implementar propuestas didácticas para la familia y la comunidad educativa de cara a la prevención e intervención de ciberbullying y grooming (Tejerina y Flores, 2007; INTECO, 2009).

Es necesario concienciar a los observadores de estas conductas el papel fundamental que juegan, pero debemos enseñarles identificarlas y ofrecerles formas sencillas y probablemente anónimas para que lo denuncien ante la más mínima sospecha. Cualquier miembro de la comunidad educativa (profesorado, alumnado, familias y personal no docente) que tenga sospechas, considere la existencia de indicios razonables o tenga conocimiento expreso de una situación de ciberacoso tiene la obligación de comunicarlo al Equipo Directivo del centro.
En los Centros educativos deben incluirse reglamentos que sancionen conductas de ciberacoso así como protocolos de actuación para prevenir, detectar y actuar en los casos que aparezca esta forma de acoso.

Sería interesante la implantación de contenidos dedicados a la prevención y actuación de este fenómeno, dentro de una nueva asignatura o bien recogida de forma tranversal a lo largo de toda la formación académica del alumno.

\section{REFERENCIAS}

Aricak, T., Siyahhan, S., Uzunhasanoglu, A., Saribeyoglu, S., Ciplak, S., Yilmaz, N., y Memmedov, C., (2008). Cyberbullying among Turkish adolescents. CyberPsychology \& Behavior, 11(3), 253-261. doi:10.1089/cpb.2007.0016.

DiBasilio, A. (2008). Reducing Bullying in Middle School Students through the Use of Student Leaders. PhD diss., Saint Xavier University and Pearson Achievement Solutions.

Equipo Multidisciplinar de Investigación sobre Cyberbullying (EMICI) (2012). Protocolo de actuación escolar ante el ciberbullying. Recuperado marzo 18, 2012 de http://www.emici. net/prot/Protocolo\%20 Ciberbullying.html

Garaigordobil, M. y Fernández-Tomé, A. (2011). CCB. Cuestionario de Cyberbullying. FOCAD Formación Continuada a Distancia. Duodécima Edición Enero-Marzo. Recuperado marzo 22, $2013 \mathrm{de}$ http://www.cop.es/focad/biblioteca.asplegios.

Garaigordobil, M. (2011). Prevalencia y consecuencias del cyberbullying: una revisión. International Journal of Psychology and Psychological Therapy, 11(2), 233-54.

Instituto Nacional de Tecnologías de la Comunicación (INTECO) (2009). Guía legal sobre ciberbullying y grooming. Observatorio de la Seguridad de la Información. Recuperado marzo 18, 2012 de http://www.inteco.es/Seguridad/Observatorio/manuales_es/guiaManual_groming_ciberbullying

Mendoza López, E. (2012) Acoso cibernético o cyberbullying: Acoso con la tecnología electrónica. Pediatría de México, 14 (3), 133-146. 
Salmivalli, C., Lagerspetz, K., Björkqvist, K., Österman, K., y Kaukiainen, A. (1996). Bullying as a group process: Participant roles and their relations to social status within the group. $\mathrm{Ag}$ gressive Behavior, 22(1), 1-15.

Salmivalli, C. (2010). Bullying and the peer group: A review. Aggression and Violent Behaviour, 15(2), 112-120.

Tejerina, O. y Flores, J. (2007). Guía para la gente legal de Internet. E-Legales. Recuperado marzo 16, 2012 de http://www.pantallasamigas.net/recursos-educativos-materialesdidacticos/e-legales-guia-para-la-gente-legalde-internet/index.htm
Urueña, A. (Coord.) (2011). Perfil sociodemográfico de los internautas. Análisis de datos INE Observatorio Nacional de las Telecomunicaciones y de la Sociedad de la Información. Recuperado marzo 21, 2012 de http://www.ontsi.red.es/ontsi/es/estudiosinformes/perfil-sociodemogr\%C3\%A1 ficode-los-internautas-an\%C3\%A1lisis-de-datos-ine-2011

Wright, V. H., Burnham, J. J., Inman, C., y Ogorchock, H. (2009). Cyberbullying: Using Virtual Scenarios to Educate and Raise Awareness. Journal of Computing in Teacher Education, 26(1), 35-42. 